Bull. Austral. Math. Soc.

Vol. 55 (1997) [29-44]

\title{
DUALITY FOR A \\ NON-DIFFERENTIABLE PROGRAMMING PROBLEM
}

J. ZHANG AND B. MOND

A generalised dual to a non-differentiable programming problem is given and duality established under general convexity and invexity conditions. A second order dual is also given and duality theorems proved under generalised second order invexity conditions.

\section{INTRODUCTION}

In [11], Mond considered the class of non-differentiable mathematical programming problems

$$
\begin{aligned}
& \text { Minimise } f(x)+\left(x^{T} B x\right)^{1 / 2} \\
& \text { subject to } g(x) \geqslant 0
\end{aligned}
$$

where $f$ and $g$ are twice differentiable functions from $R^{n}$ to $R$ and $R^{m}$ respectively, and $B$ is an $\mathrm{n} \times \mathrm{n}$ positive semi-definite (symmetric) matrix. Let $x_{0}$ satisfy (1); Mond [11] defined the set

$$
\begin{gathered}
Z_{0}=\left\{z \mid z^{T} \nabla g_{i}\left(x_{0}\right) \geqslant 0 \quad\left(\forall i \in Q_{0}\right), \quad\right. \text { and } \\
z^{T} \nabla f\left(x_{0}\right)+z^{T} B x_{0} /\left(x_{0}^{T} B x_{0}\right)^{1 / 2}<0, \text { if } x_{0}^{T} B x_{0}>0 ; \\
\left.z^{T} \nabla f\left(x_{0}\right)+\left(z^{T} B z\right)^{1 / 2}<0, \text { if } x_{0}^{T} B x_{0} \doteq 0\right\}
\end{gathered}
$$

where $Q_{0}=\left\{i \mid g_{i}\left(x_{0}\right)=0\right\}$, and established the following necessary conditions for $x_{0}$ to be an optimal solution to $(\mathrm{P})$.

PROPOSITION 1. If $x_{0}$ is an optimal solution of $(P)$ and the corresponding set $Z_{0}$ is empty, then there exist $y \in R^{m}, y \geqslant 0$, and $w \in R^{n}$ such that

$$
y^{T} g\left(x_{0}\right)=0, \quad \nabla y^{T} g\left(x_{0}\right)=\nabla f\left(x_{0}\right)+B w, \quad w^{T} B w \leqslant 1, \quad\left(x_{0}^{T} B x_{0}\right)^{1 / 2}=x_{0}^{T} B w .
$$

(Mond and Schechter [12] gave a constraint qualification which assures that $Z_{0}$ is empty. Additional constraint qualifications were given by Wolkowitz [18].)

Received 5th February, 1996

Copyright Clearance Centre, Inc. Serial-fee code: 0004-9729/97 \$A2.00+0.00. 
Using these necessary conditions, a Wolfe type dual problem [17] was formulated in [11]:

(WD)

$$
\begin{aligned}
& \text { Maximise } f(u)-y^{T} g(u)+u^{T}\left[\nabla y^{T} g(u)-\nabla f(u)\right] \\
& \text { subject to } \nabla f(u)+B w=\nabla y^{T} g(u) \\
& \qquad w^{T} B w \leqslant 1 \\
& y \geqslant 0 .
\end{aligned}
$$

(WD) is a dual to (P) assuming that $f$ is convex and $g$ is concave.

Chandra, Craven and Mond [4] weakened the convexity requirements for duality by giving a Mond-Weir type dual [14]

$$
\begin{array}{cl}
\text { Maximise } & f(u)+u^{T}\left[\nabla y^{T} g(u)-\nabla f(u)\right] \\
\text { subject to } & \nabla f(u)-\nabla y^{T} g(u)+B w=0 \\
& y^{T} g(u) \leqslant 0 \\
& w^{T} B w \leqslant 1 \\
& y \geqslant 0
\end{array}
$$

and established duality theorems assuming that $f()+.(.)^{T} B w$ is pseudo-convex for all $w \in R^{n}$ and that $y^{T} g$ is quasi-concave.

Mond and Smart [13] later generalised the results obtained by Mond [11] and Chandra, Craven and Mond [4] to invexity conditions ([3, 5, 7]). Bector and Chandra [2] recently presented two different second order duals to $(P)$, which extended the results obtained by Mangasarian [8], Mond [10] and Mond and Weir [15] for second order duality and the results obtained by Mond [11], Mond and Weir [14] and Chandra, Craven and Mond [4] for first order duality.

In this paper, we propose a general Mond-Weir type dual [14] to (P) and establish the duality theorems under both convexity and invexity conditions. A general second order Mond-Weir dual [15] to (P) will also be proposed and duality results established under generalised second order invexity conditions [1].

We shall make use of the generalised Schwarz inequality ([6] and [16])

$$
\left(x^{T} B w\right) \leqslant\left(x^{T} B x\right)^{1 / 2}\left(w^{T} B w\right)^{1 / 2}
$$

Note that equality holds if, for $\lambda \geqslant 0, B x=\lambda B w$. 


\section{DUALITY}

We propose the following general dual (GD) to (P).

$$
\begin{aligned}
\text { Maximise } & f(u)-\sum_{i \in I_{0}} y_{i} g_{i}(u)+u^{T} B w \\
\text { subject to } & \nabla f(u)-\nabla y^{T} g(u)+B w=0 \\
& \sum_{i \in I_{\alpha}} y_{i} g_{i}(u) \leqslant 0, \quad \alpha=1,2, \ldots, r \\
& w^{T} B w \leqslant 1 \\
& y \geqslant 0
\end{aligned}
$$

where $I_{\alpha} \subseteq M=\{1,2, \ldots, m\}, \quad \alpha=0,1,2, \ldots, r$ with

$$
\bigcup_{\alpha=0}^{\Gamma} I_{\alpha}=M \text { and } I_{\alpha} \cap I_{\beta}=\phi \text { if } \alpha \neq \beta
$$

Theorem 1. (Weak Duality) Let $x$ be feasible for $(P)$ and $(u, y, w)$ feasible for $(G D)$. If, for all feasible $(x, u, y, w), f()-.\sum_{i \in I_{0}} y_{i} g_{i}()+.(.)^{T} B w$ is pseudo-invex and $\sum_{i \in I_{\alpha}} y_{i} g_{i}(),. \alpha=1,2, \ldots, r$ is quasi-incave with respect to the same $\eta$, then

$$
\text { infimum }(P) \geqslant \operatorname{supremum}(G D) \text {. }
$$

Proof: Since $x$ is feasible for (P) and $(u, y, w)$ is feasible for (GD), we have

$$
\sum_{i \in I_{\alpha}} y_{i} g_{i}(x)-\sum_{i \in I_{\alpha}} y_{i} g_{i}(u) \geqslant 0, \quad \alpha=1,2, \ldots, r
$$

By the quasi-incavity of $\sum_{i \in I_{\alpha}} y_{i} g_{i}, \quad \alpha=1,2, \ldots, r$, it follows that

$$
\eta(x, u)^{T} \nabla \sum_{i \in I_{\alpha}} y_{i} g_{i}(u) \geqslant 0, \quad \alpha=1,2, \ldots, r .
$$

Hence

$$
\eta(x, u)^{T} \nabla\left(\sum_{i \in M \backslash I_{0}} y_{i} g_{i}(u)\right) \geqslant 0,
$$

then from (3), it follows that

$$
\eta(x, u)^{T}\left[\nabla f(u)-\nabla \sum_{i \in I_{0}} y_{i} g_{i}(u)+B w\right] \geqslant 0 .
$$


The pseudo-invexity of $f()-.\sum_{i \in I_{0}} y_{i} g_{i}()+.(.)^{T} B w$ then yields

$$
f(x)-\sum_{i \in I_{0}} y_{i} g_{i}(x)+x^{T} B w \geqslant f(u)-\sum_{i \in I_{0}} y_{i} g_{i}(u)+u^{T} B w
$$

Thus

$$
f(x)+x^{T} B w \geqslant f(u)-\sum_{i \in I_{0}} y_{i} g_{i}(u)+u^{T} B w, \quad \text { from } y \geqslant 0 \text { and } g(x) \geqslant 0 .
$$

Since $w^{T} B w \leqslant 1$, by the generalised Schwarz inequality (2), it follows that

$$
f(x)+\left(x^{T} B x\right)^{1 / 2} \geqslant f(u)-\sum_{i \in I_{0}} y_{i} g_{i}(u)+u^{T} B w .
$$

THEOREM 2. (Strong Duality) If $x_{0}$ is an optimal solution of $(P)$ and the corresponding set $Z_{0}$ is empty, then there exist $y \in R^{m}$ and $w \in R^{n}$ such that $\left(x_{0}, y, w\right)$ is feasible for $(G D)$ and the corresponding values of $(P)$ and $(G D)$ are equal. If, also, $f()-.\sum_{i \in I_{0}} y_{i} g_{i}()+.(.)^{T} B w$ is pseudo-invex for all $w \in R^{n}$ and $\sum_{i \in I_{\alpha}} y_{i} g_{i}(),. \alpha=1,2, \ldots, r$ is quasi-incave with respect to the same $\eta$, then $\left(x_{0}, y, w\right)$ is optimal for (GD).

Proof: Since $x_{0}$ is an optimal solution to $(\mathrm{P})$ and the corresponding set $Z_{0}$ is empty, then from Proposition 1, there exist $y \in R^{m}$ and $w \in R^{n}$ such that

$$
y^{T} g\left(x_{0}\right)=0, \nabla y^{T} g\left(x_{0}\right)=\nabla f\left(x_{0}\right)+B w, w^{T} B w \leqslant 1,\left(x_{0}^{T} B x_{0}\right)^{1 / 2}=x_{0}^{T} B w, y \geqslant 0 .
$$

So, $\left(x_{0}, y, w\right)$ is feasible for (GD) and the corresponding values of (P) and (GD) are equal. If $f()-.\sum_{i \in I_{0}} y_{i} g_{i}()+.(.)^{T} B w$ is pseudo-invex for all $w \in R^{n}$ and $\sum_{i \in I_{\alpha}} y_{i} g_{i}($. $\alpha=1,2, \ldots, r$, is quasi-incave with respect to the same $\eta$, then from Theorem 1 , $\left(x_{0}, y, w\right)$ must be an optimal solution for (GD).

We now consider some special cases of the dual (GD) and Theorems 1 and 2.

If $I_{0}=M$, then (GD) becomes (WD) and from Theorems 1 and 2, (WD) is a dual to $(\mathrm{P})$ if $f()-.y^{T} g()+.(.)^{T} B w$ is pseudo-invex with respect to $\eta$.

In the case $I_{0}=\phi$ and $I_{\alpha}=M$ (for some $\alpha \in\{1,2, \ldots, r\}$ ) then (GD) becomes (M-WD) and from Theorems 1 and 2 (M-WD) is a dual to (P) if $f()+.(.)^{T} B w$ is pseudo-invex and $y^{T} g$ is quasi-incave with respect to the same $\eta$. This extends the results obtained in [4] because pseudo-convex and quasi-concave functions are pseudoinvex and quasi-incave functions respectively. 
If $I_{0}=\phi, I_{1}=\{1\}, \ldots, I_{m}=\{m\}(r=m)$, then (GD) becomes

$$
\begin{aligned}
\text { Maximise } & f(u)+u^{T} B w \\
\text { subject to } & \nabla f(u)-\nabla y^{T} g(u)+B w=0 \\
& y_{i} g_{i}(u) \leqslant 0, \quad i=1,2, \ldots, m \\
& w^{T} B w \leqslant 1 \\
& y \geqslant 0
\end{aligned}
$$

and (M-WD1) is a dual to (P) if $f()+.(.)^{T} B w$ is pseudo-invex and each $y_{i} g_{i}, i=$ $1,2, \ldots, m$ is quasi-incave with respect to the same $\eta$. Note that if $g_{i}$ is quasi-incave with respect to $\eta, y_{i} \geqslant 0$, then $y_{i} g_{i}$ is quasi-incave with respect to the same $\eta$; thus (M-WD1) is a dual to (P) if $f()+.(.)^{T} B w$ is pseudo-invex and each $g_{i}, i=1,2, \ldots, m$ is quasi-incave with respect to the same $\eta$.

The following corollaries obviously hold because pseudo-convex and quasi-concave functions are, respectively, pseudo-invex and quasi-incave functions.

Corollary 1. (Weak Duality) Let $x$ be feasible for $(P)$ and $(u, y, w)$ feasible for (GD). If, for all feasible $(x, u, y, w), f()-.\sum_{i \in I_{0}} y_{i} g_{i}()+.(.)^{T} B w$ is pseudo-convex and $\sum_{i \in I_{\alpha}} y_{i} g_{i}(),. \alpha=1,2, \ldots, r$ is quasi-concave, then

$$
\text { infimum }(P) \geqslant \operatorname{supremum}(G D) \text {. }
$$

Corollary 2. (Strong Duality) If $x_{0}$ is an optimal solution to $(P)$ and the corresponding set $Z_{0}$ is empty, then there exist $y \in R^{m}$ and $w \in R^{n}$ such that $\left(x_{0}, y, w\right)$ is feasible for $(G D)$ and the corresponding values of $(P)$ and $(G D)$ are equal. If, also, $f()-.\sum_{i \in I_{0}} y_{i} g_{i}()+.(.)^{T} B w$ is pseudo-convex for all $w \in R^{n}$ and $\sum_{i \in I_{\alpha}} y_{i} g_{i}(),. \alpha=$ $1,2, \ldots, r$ is quasi-concave, then $\left(x_{0}, y, w\right)$ is an optimal for (GD).

Theorem 3. (Converse Duality) Let $\left(x^{*}, y^{*}, w^{*}\right)$ be optimal to (GD) at which the matrix

$$
\nabla^{2} f\left(x^{*}\right)-\nabla^{2} y^{* T} g\left(x^{*}\right)
$$

is positive or negative definite and the vectors

$$
\left\{\sum_{i \in I_{\alpha}} \nabla y_{i}^{*} g_{i}\left(x^{*}\right), \quad \alpha=1,2, \ldots, r\right\}
$$

are linearly independent. If, for all feasible $(x, u, y, w), f()-.\sum_{i \in I_{0}} y_{i} g_{i}()+.(.)^{T} B w$ is pseudo-convex and $\sum_{i \in I_{\alpha}} y_{i} g_{i}(),. \alpha=1,2, \ldots, r$ is quasi-concave, or $f()-.\sum_{i \in I_{0}} y_{i} g_{i}()+$. 
$(.)^{T} B w$ is pseudo-invex and $\sum_{i \in I_{\alpha}} y_{i} g_{i}(),. \alpha=1,2, \ldots, r$ is quasi-incave with respect to the same $\eta$, then $x^{*}$ is an optimal to $(P)$.

ProOF: Since $\left(x^{*}, y^{*}, w^{*}\right)$ is an optimal solution to (GD), by the generalised FritzJohn theorem [9], there exist $\tau_{0} \in R, \nu \in R^{n}, \tau_{\alpha} \in R, \alpha=1,2, \ldots, r, \beta \in R$ and $\gamma \in R^{m}$ such that

$$
\begin{aligned}
\tau_{0}\left(-\nabla f\left(x^{*}\right)+\sum_{i \in I_{0}} \nabla y_{i}^{*} g_{i}\left(x^{*}\right)-B w^{*}\right) & +\nu^{T}\left(\nabla^{2} f\left(x^{*}\right)-\nabla^{2} y^{* T} g\left(x^{*}\right)\right) \\
& +\sum_{\alpha=1}^{r} \tau_{\alpha}\left(\sum_{i \in I_{\alpha}} \nabla y_{i}^{*} g_{i}\left(x^{*}\right)\right)=0
\end{aligned}
$$

$$
\begin{gathered}
\tau_{0} g_{i}\left(x^{*}\right)-\nu^{T} \nabla g_{i}\left(x^{*}\right)-\gamma_{i}=0, \quad i \in I_{0} \\
\nu^{T} \nabla g_{i}\left(x^{*}\right)-\tau_{\alpha} g_{i}\left(x^{*}\right)+\gamma_{i}=0, \quad i \in I_{\alpha}, \alpha=1,2, \ldots, r, \\
\tau_{0}\left(B x^{*}\right)-\nu^{T} B-2 \beta\left(B w^{*}\right)=0 \\
\tau_{\alpha}\left(\sum_{i \in I_{\alpha}} y_{i}^{*} g_{i}\left(x^{*}\right)\right)=0, \quad \alpha=1,2, \ldots, r \\
\beta\left(w^{* T} B w^{*}-1\right)=0 \\
\gamma^{T} y^{*}=0 \\
\left(\tau_{0}, \tau_{1}, \ldots, \tau_{\alpha}, \beta, \gamma\right) \geqslant 0 \\
\left(\tau_{0}, \tau_{1}, \ldots, \tau_{\alpha}, \beta, \gamma, \nu\right) \neq 0 .
\end{gathered}
$$

Multiplying (8) by $y_{i}^{*} \geqslant 0, i \in I_{\alpha}, \alpha=1,2, \ldots, r$ and using (12) yields

$$
\nu^{T} \nabla y_{i}^{*} g_{i}\left(x^{*}\right)-\tau_{\alpha} y_{i}^{*} g_{i}\left(x^{*}\right)=0, \quad i \in I_{\alpha}, \quad \alpha=1,2, \ldots, r
$$

Hence

$$
\nu^{T} \sum_{i \in I_{\alpha}} \nabla y_{i}^{*} g_{i}\left(x^{*}\right)-\tau_{\alpha} \sum_{i \in I_{\alpha}} y_{i}^{*} g_{i}\left(x^{*}\right)=0, \quad \alpha=1,2, \ldots, r .
$$

From (10), it follows that

$$
\nu^{T} \sum_{i \in I_{\alpha}} \nabla y_{i}^{*} g_{i}\left(x^{*}\right)=0, \quad \alpha=1,2, \ldots, r
$$

Using the equation constraint (3), (6) becomes

$$
\sum_{\alpha=1}^{r}\left(\tau_{\alpha}-\tau_{0}\right)\left(\sum_{i \in I_{\alpha}} \nabla y_{i}^{*} g_{i}\left(x^{*}\right)\right)+\nu^{T}\left(\nabla^{2} f\left(x^{*}\right)-\nabla^{2} y^{* T} g\left(x^{*}\right)\right)=0
$$


Multiplying (16) by $\nu$ and using (15) gives

$$
\nu^{T}\left(\nabla^{2} f\left(x^{*}\right)-\nabla^{2} y^{* T} g\left(x^{*}\right)\right) \nu=0
$$

By assuming that $\nabla^{2} f-\nabla^{2} y^{T} g$ is positive or negative definite at $\left(x^{*}, y^{*}, w^{*}\right)$ it follows that

$$
\nu=0
$$

Then (16) gives

$$
\sum_{\alpha=1}^{r}\left(\tau_{\alpha}-\tau_{0}\right)\left(\sum_{i \in I_{\alpha}} \nabla y_{i}^{*} g_{i}\left(x^{*}\right)\right)=0
$$

Since the vectors $\left\{\sum_{i \in I_{\alpha}} \nabla y_{i}^{*} g_{i}\left(x^{*}\right), \quad \alpha=1,2, \ldots, r\right\}$ are linearly independent, (18) then yields

$$
\tau_{\alpha}-\tau_{0}=0, \quad \alpha=1,2, \ldots, r .
$$

If $\tau_{0}=0$, then $\tau_{\alpha}=0, \quad \alpha=1,2, \ldots, r$ from (19), $\gamma=0$ from (7) and (8), and $\beta=0$ from (9) and (11), but $\left(\tau_{0}, \tau_{1}, \tau_{2}, \ldots, \tau_{r}, \nu, \gamma, \beta\right)=0$ contradicts (14). So $\tau_{0}>0$. This gives $\tau_{\alpha}>0, \alpha=1,2, \ldots, r$. Then (7), (8), (13) and $\tau_{\alpha}>0, \alpha=0,1,2, \ldots, r$ yield $g\left(x^{*}\right) \geqslant 0$. Therefore, $x^{*}$ is feasible for $(\mathrm{P})$.

Multiplying (7) by $y_{i}^{*}, i \in I_{0}$ and using (12) gives

$$
\tau_{0} y_{i}^{*} g_{i}\left(x^{*}\right)=0, i \in I_{0} .
$$

Then from $\tau_{0}>0$, it follows that

$$
y_{i} g_{i}\left(x^{*}\right)=0, i \in I_{0}
$$

Also, $\nu=0, \tau_{0}>0$ and (9) give

$$
B x^{*}=\left(2 \beta / \tau_{0}\right) B w^{*}
$$

\section{Hence}

$$
\left(x^{* T} B w^{*}\right)=\left(x^{* T} B x^{*}\right)^{1 / 2}\left(w^{* T} B w^{*}\right)^{1 / 2} .
$$

If $\beta>0$, then (11) gives $w^{* T} B w^{*}=1$ and so (22) yields

$$
\left(x^{* T} B w^{*}\right)=\left(x^{* T} B x^{*}\right)^{1 / 2} .
$$


If $\beta=0$ then (21) gives $B x^{*}=0$. So we still get

$$
\left(x^{* T} B w^{*}\right)=\left(x^{* T} B x^{*}\right)^{1 / 2} \text {. }
$$

Thus in either case, we obtain

$$
\left(x^{* T} B w^{*}\right)=\left(x^{* T} B x^{*}\right)^{1 / 2} .
$$

Therefore from (20) and (23), we have

$$
f\left(x^{*}\right)+\left(x^{* T} B x^{*}\right)^{1 / 2}=f\left(x^{*}\right)-\sum_{i \in I_{0}} y_{i}^{*} g_{i}\left(x^{*}\right)+x^{* T} B w^{*} .
$$

If, for all feasible $(x, u, y, w), f()-.\sum_{i \in I_{0}} y_{i} g_{i}()+.(.)^{T} B w$ is pseudo-convex and $\sum_{i \in I_{\alpha}} y_{i} g_{i}($.$) ,$ $\alpha=1,2, \ldots, r$ is quasi-concave, or $f()-.\sum_{i \in I_{0}} y_{i} g_{i}()+.(.)^{T} B w$ is pseudo-invex and $\sum_{i \in I_{\alpha}} y_{i} g_{i}(),. \alpha=1,2, \ldots, r$ is quasi-incave with respect to the same $\eta$, then fromTheorem 1 or Corollary $1, x^{*}$ is an optimal solution to $(\mathrm{P})$.

\section{SECOND ORDER DUALITY}

In this section, we present a general non-differentiable second order Mond-Weir dual $[15]$ to $(\mathrm{P})$. We shall make use of the following definitions.

Definition 1: [1] $f$ is second order pseudo-invex if for all $p \in R^{n}$, there exists an $\eta(x, u)$ such that

$$
\eta(x, u)^{T}\left[\nabla f(u)+\nabla^{2} f(u) p\right] \geqslant 0 \Longrightarrow f(x) \geqslant f(u)-\frac{1}{2} p^{T} \nabla^{2} f(u) p .
$$

Definition 2: [1] $f$ is second order quasi-invex if for all $p \in R^{n}$, there exists an $\eta(x, u)$ such that

$$
f(x) \leqslant f(u)-\frac{1}{2} p^{T} \nabla^{2} f(u) p \Longrightarrow \eta(x, u)^{T}\left[\nabla f(u)+\nabla^{2} f(u) p\right] \leqslant 0 .
$$

A function $g$ is said to be second order pseudo-incave or second order quasi-incave if $-\boldsymbol{g}$ is second order pseudo-invex and second order qausi-invex respectively.

The second order Mangasarian type [8] and Mond-Weir type [15] duals to $(P)$ were regarded in $[2]$ as the following problems:

$$
\begin{array}{cl}
\text { Maximise } & f(u)-y^{T} g(u)+u^{T} B w-\frac{1}{2} p^{T} \nabla^{2}\left[f(u)-y^{T} g(u)\right] p \\
\text { subject to } & \nabla f(u)-\nabla y^{T} g(u)+B w+\nabla^{2} f(u) p-\nabla^{2} y^{T} g(u) p=0 \\
& w^{T} B w \leqslant 1 \\
& y \geqslant 0
\end{array}
$$


where $u, w, p, \in R^{n}$ and $y \in R^{m}$.

(2M-WD)

$$
\begin{array}{ll}
\text { Maximise } & f(u)+u^{T} B w-\frac{1}{2} p^{T} \nabla^{2} f(u) p \\
\text { subject to } & \nabla f(u)-\nabla y^{T} g(u)+B w+\nabla^{2} f(u) p-\nabla^{2} y^{T} g(u) p=0 \\
& y^{T} g(u)-\frac{1}{2} p^{T} \nabla^{2} y^{T} g(u) p \leqslant 0 \\
& w^{T} B w \leqslant 1 \\
& y \geqslant 0
\end{array}
$$

Using the second order convexity conditions (called bonvexity in [2]), Bector and Chandra established duality theorems between (P) and (2MD) and (2M-WD), respectively.

Following Mond-Weir [15] we now propose a general second order dual (2GD) to (P)

(2GD)

$$
\text { Maximise } f(u)-\sum_{i \in I_{0}} y_{i} g_{i}(u)+u^{T} B w-\frac{1}{2} p^{T}\left[\nabla^{2} f(u)-\nabla^{2} \sum_{i \in I_{0}} y_{i} g_{i}(u)\right] p
$$

$$
\begin{array}{ll}
\text { subject to } & \nabla f(u)-y^{T} g(u)+B w+\nabla^{2} f(u) p-\nabla^{2} y^{T} g(u) p=0 \\
& \sum_{i \in I_{\alpha}} y_{i} g_{i}(u)-\frac{1}{2} p^{T} \nabla^{2} \sum_{i \in I_{\alpha}} y_{i} g_{i}(u) p \leqslant 0, \alpha=1,2, \ldots, r, \\
& w^{T} B w \leqslant 1 \\
& y \geqslant 0
\end{array}
$$

where $I_{\alpha} \subseteq M=\{1,2, \ldots, m\}, \alpha=0,1,2, \ldots, r$ with

$$
\bigcup_{\alpha=0}^{r} I_{\alpha}=M \text { and } I_{\alpha} \cap I_{\beta}=\phi \text { if } \alpha \neq \beta
$$

Theorem 4. (Weak Duality) Let $x$ be feasible for $(P)$ and $(u, y, w, p)$ feasible for (2GD). If, for all feasible $(x, u, y, w, p), f()-.\sum_{i \in I_{0}} y_{i} g_{i}()+.(.)^{T} B w$ is second order pseudo-invex, and $\sum_{i \in I_{\alpha}} y_{i} g_{i}(),. \alpha=1,2, \ldots, r$ is second order quasi-incave with respect to the same $\eta$, then

$$
\operatorname{infimum}(P) \geqslant \operatorname{supremum}(2 G D) \text {. }
$$


Proof: Since $x$ is feasible for (P) and $(u, y, w, p)$ is feasible for (2GD), we have

$$
\sum_{i \in I_{\alpha}} y_{i} g_{i}(x)-\sum_{i \in I_{\alpha}} y_{i} g_{i}(u)-\frac{1}{2} p^{T} \nabla^{2} \sum_{i \in I_{\alpha}} y_{i} g_{i}(u) p \geqslant 0, \quad \alpha=1,2, \ldots, r .
$$

By the second order quasi-incavity of $\sum_{i \in I_{\alpha}} y_{i} g_{i}, \alpha=1,2, \ldots, r$, it follows that

$$
\eta(x, u)^{T}\left(\nabla \sum_{i \in I_{\alpha}} y_{i} g_{i}(u)+\nabla^{2} \sum_{i \in I_{\alpha}} y_{i} g_{i}(u) p\right) \geqslant 0, \quad \alpha=1,2, \ldots, r
$$

Hence

$$
\eta(x, u)^{T}\left(\nabla \sum_{i \in M \backslash I_{0}} y_{i} g_{i}(u)+\nabla^{2} \sum_{i \in M \backslash I_{0}} y_{i} g_{i}(u) p\right) \geqslant 0 .
$$

Then from (24), (28) yields

$$
\eta(x, u)^{T}\left(\nabla f(u)+\nabla^{2} f(u) p-\sum_{i \in I_{0}} y_{i} g_{i}(u)-\nabla^{2} \sum_{i \in I_{0}} y_{i} g_{i}(u) p+B w\right) \geqslant 0 .
$$

Since $f()-.\sum_{i \in I_{0}} y_{i} g_{i}()+.(.)^{T} B w$ is second order pseudo-invex, it follows that

$$
\begin{aligned}
& f(x)-\sum_{i \in I_{0}} y_{i} g_{i}(x)+x^{T} B w \\
& \quad \geqslant f(u)-\sum_{i \in I_{0}} y_{i} g_{i}(u)+u^{T} B w-\frac{1}{2} p^{T} \nabla^{2}\left[f(u)-\sum_{i \in I_{0}} y_{i} g_{i}(u)+u^{T} B w\right] p
\end{aligned}
$$

Thus, from $y \geqslant 0, g(x) \geqslant 0$, we have

(29) $f(x)+x^{T} B w \geqslant f(u)-\sum_{i \in I_{0}} y_{i} g_{i}(u)+u^{T} B w-\frac{1}{2} p^{T}\left[\nabla^{2} f(u)-\nabla^{2} \sum_{i \in I_{0}} y_{i} g_{i}(u)\right] p$.

Since $\boldsymbol{w}^{T} B w \leqslant 1$, by the generalised Schwarz inequality (2), (29) gives that

$$
f(x)+\left(x^{T} B x\right)^{1 / 2} \geqslant f(u)-\sum_{i \in I_{0}} y_{i} g_{i}(u)+u^{T} B w-\frac{1}{2} p^{T}\left[\nabla^{2} f(u)-\nabla^{2} \sum_{i \in I_{0}} y_{i} g_{i}(u)\right] p .
$$

THEOREM 5. (Strong Duality) If $x_{0}$ is an optimal solution to $(P)$ and the corresponding set $Z_{0}$ is empty, then there exist $y \in R^{m}$ and $w \in R^{n}$ such that $\left(x_{0}, y, w, p=0\right)$ is feasible for (2GD), and the corresponding values of $(P)$ and $(2 G D)$ are 
equal. If, for all feasible $(x, u, y, p, w), f()-.\sum_{i \in I_{0}} y_{i} g_{i}()+.(.)^{T} B w$ is second order pseudoinvex, and $\sum_{i \in I_{\alpha}} y_{i} g_{i}(),. \alpha=1,2, \ldots, r$ is second order quasi-incave, then $\left(x_{0}, y, w, p=0\right)$ is an optimal solution for (2GD).

Proof: Since $x_{0}$ is an optimal solution to $(\mathrm{P})$ and the corresponding set $Z_{0}$ is empty, then from Proposition 1, there exist $y \in R^{m}$ and $w \in R^{n}$ such that

$$
\begin{aligned}
y^{T} g\left(x_{0}\right)=0, \quad \nabla y^{T} g\left(x_{0}\right) & =\nabla f\left(x_{0}\right)+B w, \quad w^{T} B w \leqslant 1, \\
\left(x_{0}^{T} B x_{0}\right)^{1 / 2} & =x_{0}^{T} B w, \quad y \geqslant 0 .
\end{aligned}
$$

So, $\left(x_{0}, y, w, p=0\right)$ is feasible for (2GD) and the corresponding values of (P) and (2GD) are equal. If $f()-.\sum_{i \in I_{0}} y_{i} g_{i}()+.(.)^{T} B w$ is second order pseudo-invex for all $w \in R^{n}$ and $\sum_{i \in I_{\alpha}} y_{i} g_{i}(),. \alpha=1,2, \ldots, r$, is second order quasi-incave with respect to the same $\eta$, then from Theorem $4,\left(x_{0}, y, w, p=0\right)$ must be an optimal solution for (2GD).

We now consider some special cases of (2GD) and Theorems 4 and 5.

If $I_{0}=M$, then (2GD) becomes (2MD), and from Theorems 4 and 5, (2MD) is a second order dual to (P) if $f()-.y^{T} g()+.(.)^{T} B w$ is second order pseudo-invex, which extends the results obtained in [2] because second order pseudo-convex and second order quasi-concave are second order pseudo-invex and second order quasi-incave respectively [1].

If $I_{0}=\phi$ and $I_{\alpha}=M$ (for some $\alpha \in\{1,2, \ldots, r\}$ ), then (2GD) becomes (2M$\mathrm{WD}$ ), and from Theorems 4 and $5,(2 \mathrm{M}-\mathrm{WD})$ is a second order dual to (P) if $f()+.(.)^{T} B w$ is second order pseudo-invex and $y^{T} g$ is second order quasi-incave, which extends the results obtained in [2].

We now assume that $f$ and $g$ are three times differentiable.

THEOREM 6. (Converse Duality) Let $\left(x^{*}, y^{*}, w^{*}, p^{*}\right)$ be an optimal solution to (2GD) at which the matrix

$$
\nabla\left[\nabla^{2} f\left(x^{*}\right)-\nabla^{2} y^{* T} g\left(x^{*}\right)\right] p^{*}
$$

is positive or negative definite and the vectors

$$
\left\{\left[\nabla^{2} f\left(x^{*}\right)-\nabla^{2} \sum_{i \in I_{0}} y_{i}^{*} g_{i}\left(x^{*}\right)\right]_{j}, \quad\left[\nabla^{2} \sum_{i \in I_{\alpha}} y_{i}^{*} g_{i}\left(x^{*}\right)\right]_{j}, \quad \alpha=1,2, \ldots, r, \quad j=1,2, \ldots, n\right\}
$$

are linearly independent, where $\left[\nabla^{2} f-\nabla^{2} \sum_{i \in I_{0}} y_{i} g_{i}\right]_{j}$ is the $j$-th row of $\nabla^{2} f-$ $\nabla^{2} \sum_{i \in I_{0}} y_{i} g_{i}$ and $\left[\nabla^{2} \sum_{i \in I_{\alpha}} y_{i} g_{i}\right]_{j}$ is the $j$-th row of $\nabla^{2} \sum_{i \in I_{\alpha}}\left(y_{i} g_{i}\right)$. If, for all feasible 
$(x, u, y, w, p), f()-.\sum_{i \in I_{0}} y_{i} g_{i}()+.(.)^{T} B w$ is second order pseudo-invex and $\sum_{i \in I_{\alpha}} y_{i} g_{i}($.$) ,$ $\alpha=1,2, \ldots, r$ is second order quasi-incave with respect to the same $\eta$, then $x^{*}$ is an optimal solution to $(P)$.

PROOF: Since $\left(x^{*}, y^{*}, w^{*}, p^{*}\right)$ is an optimal solution to (2GD), by the generalised Fritz-John theorem [9], there exist $\tau_{0} \in R, \nu \in R^{n}, \tau_{\alpha} \in R, \alpha=1,2, \ldots, r, \beta \in R$ and $\gamma \in R^{m}$ such that

$$
\begin{gathered}
\tau_{0}\left\{-\nabla f\left(x^{*}\right)+\sum_{i \in I_{0}} \nabla y_{i}^{*} g_{i}\left(x^{*}\right)-B w^{*}+\frac{1}{2} p^{* T} \nabla\left[\nabla^{2} f\left(x^{*}\right)-\nabla^{2} \sum_{i \in I_{0}} y_{i}^{*} g_{i}\left(x^{*}\right) p^{*}\right]\right\} \\
+\nu^{T}\left\{\nabla^{2} f\left(x^{*}\right)-\nabla^{2} y^{* T} g\left(x^{*}\right)+\nabla\left[\nabla^{2} f\left(x^{*}\right) p^{*}-\nabla^{2} y^{* T} g\left(x^{*}\right) p^{*}\right]\right\}
\end{gathered}
$$

$$
+\sum_{\alpha=1}^{r} \tau_{\alpha}\left\{\nabla \sum_{i \in I_{\alpha}} y_{i}^{*} g_{i}\left(x^{*}\right)-\frac{1}{2} p^{* T} \nabla\left[\nabla^{2} \sum_{i \in I_{\alpha}} y_{i}^{*} g_{i}\left(x^{*}\right) p^{*}\right]\right\}=0
$$

$$
\begin{gathered}
\tau_{0}\left\{g_{i}\left(x^{*}\right)-\frac{1}{2} p^{* T} \nabla^{2} g_{i}\left(x^{*}\right) p^{*}\right\}-\nu^{T}\left\{\nabla g\left(x^{*}\right)+\nabla^{2} g\left(x^{*}\right) p^{*}\right\}-\gamma_{i}=0, \quad i \in I_{0}, \\
-\nu^{T}\left\{\nabla g\left(x^{*}\right)+\nabla^{2} g\left(x^{*}\right) p^{*}\right\}+\tau_{\alpha}\left\{g_{i}\left(x^{*}\right)-\frac{1}{2} p^{* T} \nabla^{2} g_{i}\left(x^{*}\right) p^{*}\right\}-\gamma_{i}=0 \\
i \in I_{\alpha}, \quad \alpha=1,2, \ldots, r \\
\tau_{0} B x^{*}-\nu^{T} B-2 \beta\left(B w^{*}\right)=0
\end{gathered}
$$

$\left(\tau_{0} p^{*}+\nu\right)^{T}\left\{\nabla^{2} f\left(x^{*}\right)-\nabla^{2} \sum_{i \in I_{0}} y_{i}^{*} g_{i}\left(x^{*}\right)\right\}-\sum_{\alpha=1}^{r}\left(\tau_{\alpha} p^{*}+\nu\right)^{T}\left\{\nabla^{2} \sum_{i \in I_{\alpha}} y_{i}^{*} g_{i}\left(x^{*}\right)\right\}=0$

$$
\begin{gathered}
\tau_{\alpha}\left\{\sum_{i \in I_{\alpha}} y_{i}^{*} g_{i}\left(x^{*}\right)-\frac{1}{2} p^{* T} \nabla^{2} \sum_{i \in I_{\alpha}} y_{i}^{*} g_{i}\left(x^{*}\right) p^{*}\right\}=0, \quad \alpha=1,2, \ldots, r \\
\beta\left(w^{* T} B w^{*}-1\right)=0 \\
\gamma^{T} y^{*}=0 \\
\left(\tau_{0}, \tau_{1}, \ldots, \tau_{r}, \beta, \gamma\right) \geqslant 0 \\
\left(\tau_{0}, \tau_{1}, \ldots, \tau_{r}, \beta, \gamma, \nu\right) \neq 0
\end{gathered}
$$

Since

$$
\left\{\left[\nabla^{2} f(u)-\nabla^{2} \sum_{i \in I_{0}} y_{i} g_{i}(u)\right]_{j}, \quad\left[\nabla^{2} \sum_{i \in I_{\alpha}} y_{i} g_{i}(u)\right]_{;}, \quad \alpha=1,2, \ldots, r, \quad j=1,2, \ldots, n\right\}
$$

are linearly independent at $\left(x^{*}, y^{*}, w^{*}, p^{*}\right),(34)$ then gives

$$
\tau_{\alpha} p^{*}+\nu=0, \quad \alpha=0,1,2, \ldots, r .
$$


Multiplying (32) by $y_{i}^{*}, i \in I_{\alpha}, \alpha=1,2, \ldots, r$ and using (37) yields

$$
\begin{gathered}
\nu^{T}\left\{\nabla y_{i}^{*} g_{i}\left(x^{*}\right)+\nabla^{2} y_{i}^{*} g_{i}\left(x^{*}\right) p^{*}\right\}-\tau_{\alpha}\left\{y_{i}^{*} g_{i}\left(x^{*}\right)-\frac{1}{2} p^{* T} \nabla^{2} y_{i}^{*} g_{i}\left(x^{*}\right) p^{*}\right\}=0 \\
i \in I_{\alpha}, \quad \alpha=1,2, \ldots, r
\end{gathered}
$$

thus

$$
\begin{aligned}
\nu^{T}\left\{\sum_{i \in I_{\alpha}} \nabla y_{i}^{*} g_{i}\left(x^{*}\right)+\sum_{i \in I_{\alpha}} \nabla^{2} y_{i}^{*} g_{i}\left(x^{*}\right) p^{*}\right\} \\
-\tau_{\alpha}\left\{\sum_{i \in I_{\alpha}} y_{i}^{*} g_{i}\left(x^{*}\right)-\frac{1}{2} p^{* T} \nabla^{2} \sum_{i \in I_{\alpha}} y_{i}^{*} g_{i}\left(x^{*}\right) p^{*}\right\}=0, \quad \alpha=1,2, \ldots, r .
\end{aligned}
$$

From (35), it follows that

$$
\nu^{T}\left\{\sum_{i \in I_{\alpha}} \nabla y_{i}^{*} g_{i}\left(x^{*}\right)+\sum_{i \in I_{\alpha}} \nabla^{2} y_{i}^{*} g_{i}\left(x^{*}\right) p^{*}\right\}=0, \quad \alpha=1,2, \ldots, r
$$

Using (24), (30) gives

$$
\begin{aligned}
\left(\tau_{\alpha} p^{*}+\nu\right)^{T} & \left\{\nabla^{2} f\left(x^{*}\right)-\nabla^{2} \sum_{i \in I_{0}} y_{i}^{*} g_{i}\left(x^{*}\right)+\nabla\left[\nabla^{2} f\left(x^{*}\right)-\nabla^{2} \sum_{i \in I_{0}} y_{i}^{*} g_{i}\left(x^{*}\right)\right] p^{*}\right\} \\
& -\sum_{\alpha=1}^{r}\left(\tau_{\alpha} p^{*}+\nu\right)^{T}\left\{\nabla^{2}\left[\sum_{i \in I_{\alpha}} y_{i}^{*} g_{i}\left(x^{*}\right)\right]+\nabla\left[\nabla^{2} \sum_{i \in I_{\alpha}} y_{i}^{*} g_{i}\left(x^{*}\right)\right] p^{*}\right\} \\
& -\tau_{0}\left\{\nabla \sum_{i \in M \backslash I_{0}} y_{i}^{*} g_{i}\left(x^{*}\right)+\nabla^{2} \sum_{i \in M \backslash I_{0}} y_{i}^{*} g_{i}\left(x^{*}\right) p^{*}\right\} \\
& -\frac{1}{2} \tau_{0} p^{* T}\left\{\nabla\left[\nabla^{2} f\left(x^{*}\right)-\nabla^{2} \sum_{i \in I_{0}} y_{i}^{*} g_{i}\left(x^{*}\right)\right] p^{*}\right\} \\
& +\sum_{\alpha=1}^{r} \tau_{\alpha}\left\{\nabla \sum_{i \in I_{\alpha}} y_{i}^{*} g_{i}\left(x^{*}\right)+\nabla^{2}\left[\sum_{i \in I_{\alpha}} y_{i}^{*} g_{i}\left(x^{*}\right)\right] p^{*}\right\} \\
& +\sum_{\alpha=1}^{r} \frac{1}{2} \tau_{\alpha} p^{* T}\left\{\nabla\left[\nabla^{2} \sum_{i \in I_{\alpha}} y_{i}^{*} g_{i}\left(x^{*}\right)\right] p^{*}\right\}=0 .
\end{aligned}
$$

From (40), it follows that

$$
\begin{aligned}
\sum_{\alpha=1}^{r}\left(\tau_{\alpha}\right. & \left.-\tau_{0}\right)\left\{\nabla \sum_{i \in I_{\alpha}} y_{i}^{*} g_{i}\left(x^{*}\right)+\nabla^{2} \sum_{i \in I_{\alpha}} y_{i}^{*} g_{i}\left(x^{*}\right) p^{*}\right\} \\
& +\frac{1}{2} \nu^{T}\left\{\nabla\left[\nabla^{2} f\left(x^{*}\right)-\nabla^{2} \sum_{i \in I_{0}} y_{i}^{*} g_{i}\left(x^{*}\right)\right] p^{*}-\nabla\left[\nabla^{2} \sum_{i \in M \backslash I_{0}} y_{i}^{*} g_{i}\left(x^{*}\right)\right] p^{*}\right\}=0
\end{aligned}
$$


That is

$$
\begin{aligned}
\sum_{\alpha=1}^{r}\left(\tau_{\alpha}-\tau_{0}\right)\left\{\nabla \sum_{i \in I_{\alpha}} y_{i}^{*} g_{i}\left(x^{*}\right)+\right. & \left.\nabla^{2} \sum_{i \in I_{\alpha}} y_{i}^{*} g_{i}\left(x^{*}\right) p^{*}\right\} \\
& +\frac{1}{2} \nu^{T}\left\{\nabla\left[\nabla^{2} f\left(x^{*}\right)-\nabla^{2} y^{* T} g\left(x^{*}\right)\right] p^{*}\right\}=0 .
\end{aligned}
$$

Multiplying (42) by $\nu$ and using (41) yields

$$
\nu^{T}\left\{\nabla\left[\nabla^{2} f\left(x^{*}\right)-\nabla^{2} y^{* T} g\left(x^{*}\right)\right] p^{*}\right\} \nu=0 .
$$

By assuming that $\nabla\left[\nabla^{2} f\left(x^{*}\right)-\nabla^{2} y^{* T} g\left(x^{*}\right)\right] p^{*}$ is positive or negative definite, it follows that

$$
\nu=0
$$

so (40) becomes

$$
\tau_{\alpha} p^{*}=-\nu=0, \quad \alpha=0,1,2, \ldots, r .
$$

If $\tau_{\alpha}=0, \alpha=0,1,2, \ldots, r$, we get $\gamma=0$ from (31) and (32), and $\beta=0$ from (33) and (36); but $\left(\tau_{0}, \tau_{1}, \ldots, \tau_{r}, \beta, \gamma, \nu\right)=0$ contradicts (39). Thus $\tau_{\alpha}>0, \alpha=0,1,2, \ldots, r$; this gives $p^{*}=0$. Hence from (31) and (32), it follows that

$$
\begin{gathered}
\tau_{0} g_{i}\left(x^{*}\right)-\gamma_{i}=0, \quad i \in I_{0} \\
\tau_{\alpha} g_{i}\left(x^{*}\right)-\gamma_{i}=0, \quad i \in I_{\alpha}, \quad \alpha=1,2, \ldots, r .
\end{gathered}
$$

Therefore $g\left(x^{*}\right) \geqslant 0$ since $\gamma \geqslant 0$ and $\tau_{\alpha}>0, \alpha=0,1,2, \ldots, r$. Thus $x^{*}$ is feasible for (P).

Multiplying (44) by $y_{i}, i \in I_{0}$ and using (37) gives

$$
\tau_{0} y_{i}^{*} g_{i}\left(x^{*}\right)=0, \quad i \in I_{0} .
$$

By $\tau_{0}>0$, it follows that

$$
y_{i}^{*} g_{i}\left(x^{*}\right)=0, \quad i \in I_{0}
$$

Also, $\nu=0, \tau_{0}>0$ and (33) give

$$
B x^{*}=\left(2 \beta / \tau_{0}\right) B w^{*} .
$$

Hence

$$
\left(x^{* T} B w^{*}\right)=\left(x^{* T} B x^{*}\right)^{1 / 2}\left(w^{* T} B w^{*}\right)^{1 / 2}
$$


If $\beta>0$, then (36) gives $w^{* T} B w^{*}=1$, and so (48) yields

$$
\left(x^{* T} B w^{*}\right)=\left(x^{* T} B x^{*}\right)^{1 / 2} \text {. }
$$

If $\beta=0$, then (47) gives $B x^{*}=0$. So we still get

$$
\left(x^{* T} B w^{*}\right)=\left(x^{* T} B x^{*}\right)^{1 / 2} .
$$

Thus, in either case, we have

$$
\left(x^{* T} B w^{*}\right)=\left(x^{* T} B x^{*}\right)^{1 / 2}
$$

Therefore from (46), (49) and $p^{*}=0$, we have

$f\left(x^{*}\right)+\left(x^{* T} B x^{*}\right)^{1 / 2}=f\left(x^{*}\right)-\sum_{i \in I_{0}} y_{i}^{*} g_{i}\left(x^{*}\right)+x^{* T} B w^{*}-\frac{1}{2} p^{* T} \nabla^{2}\left[f\left(x^{*}\right)-y^{* T} g\left(x^{*}\right)\right] p^{*}$.

If, for all feasible $(x, u, y, w, p), f()-.\sum_{i \in I_{0}} y_{i} g_{i}()+.(.)^{T} B w$ is second order pseudo-invex and $\sum_{i \in I_{\alpha}} y_{i} g_{i}(),. \alpha=1,2, \ldots, r$ is second order quasi-incave with respect to the same $\eta$, then from Theorem $4, x^{*}$ is an optimal solution to (P).

Note that if $p=0$, then (2GD) becomes (GD), (2MD) becomes (MD) and (2MWD) becomes (M-WD). This means that second order duality implies first order duality, and so Theorems 4, 5 and 6 imply Theorems 1,2 and 3 respectively.

\section{REFERENCES}

[1] C.R. Bector and B.K. Bector, '(Generalized)-binvex functions and second order duality for a nonlinear programming problem', Congr. Numer. 52 (1986), 37-52.

[2] C.R. Bector and S. Chandra, 'Second order duality with nondifferential functions', (in preparation).

[3] A. Ben-Israel and B. Mond, 'What is invexity?', J. Austral. Math. Soc. Ser. B 28 (1986), 1-9.

[4] S. Chandra, B.D. Craven and B. Mond, 'Generalized concavity and duality with a square root term', Optimization 16 (1985), 653-662.

[5] B.D. Craven, 'Duality for generalized convex fractional programs', in Generalized concavity in optimization and economics, (S. Schaible and W.T. Ziemba, Editors) (Academic Press, New York, 1981), pp. 473-489.

[6] E. Eisenberg, 'Supports of a convex function', Bull. Amer. Math. Soc. 68 (1962), 192-195.

[7] M.A. Hanson, 'On sufficiency of the Kuhn-Tucker conditions', J. Math. Anal. Appl. 80 (1981), 545-550. 
[8] O.L. Mangasarian, 'Second and higher-order duality in nonlinear programming', J. Math. Anal. Appl. 51 (1975), 607-620.

[9] O.L. Mangasarian and S. Fromovitz, 'The Fritz John necessary optimality conditions in the presence of equality and inequality conditions', J. Math. Anal. Appl. 17 (1967), 37-47.

[10] B. Mond, 'Second order duality for nonlinear programs', Opsearch 11 (1974), 90-99.

[11] B. Mond, 'A class of nondifferentiable mathematical programming problems', J. Math. Anal. Appl. 46 (1974), 169-174.

[12] B. Mond and $M$. Schechter, 'On a constraint qualification in a nondifferentiable programming problem', Naval Res. Logist. Quart. 23 (1976), 611-613.

[13] B. Mond and I. Smart, 'Duality with invexity for a class of nondifferentiable static and continuous programming problems', J. Math. Anal. Appl. 141 (1989), 373-388.

[14] B. Mond and T. Weir, 'Generalized concavity and duality', in Generalized concavity in optimization and economics, (S. Schaible and W. T. Ziemba, Editors) (Academic Press, New York, 1981), pp. 263-279.

[15] B. Mond and T. Weir, 'Generalized convexity and higher order duality', J. Math. Sci. 16-18 (1981-1983), 74-94.

[16] F. Riesz and B. Sz.-Nagy, Functional analysis, (translated from the 2nd French edition by Leo F. Boron) (Frederick Ungar Publishing Co., New York, 1955).

[17] P. Wolfe, 'A duality theorem for nonlinear programming', Quart. Appl. Math. 19 (1961), 239-244.

[18] H. Wolkowitz, 'An optimality condition for a nondifferentiable convex program', Naval Res. Logist. Quart. 30 (1983), 415-418.

School of Mathematics

La Trobe University

Bundoora Vic 3083

Australia

e-mail: b.mond@latrobe.edu.au 